Review Article

\title{
Periodontal Pathogens and Atherosclerosis: Implications of Inflammation and Oxidative Modification of LDL
}

\author{
Tomoko Kurita-Ochiai and Masafumi Yamamoto \\ Department of Microbiology and Immunology, Nihon University School of Dentistry at Matsudo, Matsudo, Chiba 271-8587, Japan \\ Correspondence should be addressed to Tomoko Kurita-Ochiai; ochiai.tomoko@nihon-u.ac.jp
}

Received 5 February 2014; Revised 7 April 2014; Accepted 28 April 2014; Published 18 May 2014

Academic Editor: Tomasz Guzik

Copyright (C) 2014 T. Kurita-Ochiai and M. Yamamoto. This is an open access article distributed under the Creative Commons Attribution License, which permits unrestricted use, distribution, and reproduction in any medium, provided the original work is properly cited.

\begin{abstract}
Inflammation is well accepted to play a crucial role in the development of atherosclerotic lesions, and recent studies have demonstrated an association between periodontal disease and cardiovascular disease. Porphyromonas gingivalis and Aggregatibacter actinomycetemcomitans, causative agents of destructive chronic inflammation in the periodontium, can accelerate atheroma deposition in animal models. Emerging evidence suggests that vaccination against virulence factors of these pathogens and antiinflammatory therapy may confer disease resistance. In this review, we focus on the role of inflammatory mechanisms and oxidative modification in the formation and activation of atherosclerotic plaques accelerated by P. gingivalis or A. actinomycetemcomitans in an ApoE-deficient mouse model and high-fat-diet-fed mice. Furthermore, we examine whether mucosal vaccination with a periodontal pathogen or the anti-inflammatory activity of catechins can reduce periodontal pathogen-accelerated atherosclerosis.
\end{abstract}

\section{Introduction}

Periodontitis, a chronic, destructive condition affecting a large portion of the adult population, is one of the major causes of tooth loss and characterized by a chronic infection associated with gram-negative anaerobic bacteria in the dental biofilm. It leads to irreversible destruction of tissues supporting the teeth and is clinically detectable as periodontal pockets and alveolar bone loss $[1,2]$. Since periodontitis often causes bacteremia, a relationship between periodontitis and systemic diseases via periodontal pathogens has been explored [3]. Two or more periodontopathic bacteria have been detected in the cardiac valve $[4,5]$ and aortic aneurysm [6]; therefore, periodontal infection could also affect the progression of cardiovascular disease (CVD). However, many questions regarding this causal relationship and pathological mechanism need to be answered.

This review discusses the involvement of periodontopathic bacteria in the development of atherosclerosis. Furthermore, we mention the possibility of preventing atherosclerosis by developing a vaccine for specific bacteria or common antigens, as well as by the intake of catechin, which has both antioxidative and anti-inflammatory effects.

\section{The Periodontal-Systemic Relationship}

Epidemiological studies have suggested that periodontal infections are associated with an increased risk of CVD [710]. For example, it was shown that patients with periodontitis have a $19 \%$ greater risk of CVD compared to subjects without periodontitis [11, 12]. Furthermore, severe periodontitis is associated with increased intima-media thickening [13], while a systemic antibody response to a periodontal organism is associated with coronary heart disease [14]. Although observational studies suggest that such an association is independent of known confounders such as hypertension, smoking, diabetes, and obesity, this contention has not been confirmed [2]. In contrast, a recent cross-sectional study indicated that periodontitis is associated with endothelial dysfunction in a general population [15].

Considerable evidence indicates that periodontopathic bacteria may directly or indirectly contribute to cardiovascular disease, such as blood platelet aggregation, enhanced lowdensity cholesterol and lipoprotein deposition in the arterial walls, invasion of cardiac and carotid endothelium, and the high level of circulating- or tissue-derived inflammatory mediators [16-18]. Periodontal bacteria may directly infect 
atherosclerotic lesions, thus contributing to the inflammatory process. The fact that periodontopathic bacteria were detected from atheromatous plaques of coronary arteries suggested that periodontal bacteria such as Porphyromonas gingivalis and Aggregatibacter actinomycetemcomitans invade endothelial cells and induce chronic vascular inflammation [19]. Furthermore, P. gingivalis, A. actinomycetemcomitans, Fusobacterium nucleatum, and other oral anaerobes were shown to degrade immunoglobulin, inhibit the complement system, and produce toxic components such as lipopolysaccharide (endotoxins) and secreted molecules (exotoxins) $[20,21]$. These factors sustain bacterial viability during bacteremia, which commonly occurs as a result of surgical treatments, tooth brushing, and other dental procedures [16, 22]. Chronic periodontal infections, initiated by gram-negative tooth-associated microbial biofilms, may also indirectly induce endothelial activation or dysfunction through a state of systemic inflammation as evidenced by elevated plasma acute phase proteins; proinflammatory cytokines such as interleukin-1 (IL-1), IL-6, and tumor necrosis factor- $\alpha$; and the matrix metalloproteinase (MMP) family [23-26]. Moreover, the release of bacterial products such as outer membrane vesicles [27] or gingipains [28] from $P$. gingivalis or the release of free soluble components from A. actinomycetemcomitans [29] into the circulation can induce proatherogenic responses in endothelial cells. Immune activation by the pathogenderived heat-shock protein (HSP) GroEL may also result in an autoimmune response followed by atherosclerosis via the structural similarity or "molecular mimicry" between host HSP60 and GroEL. Previous studies have shown that HSP60 is selectively located in atherosclerotic lesions rather than nonatherosclerotic areas of the arterial wall [30]. In addition, a significant correlation has been observed between antiHSP antibody (Ab) levels and the severity of atherosclerosis. High titers of anti-HSP60 Abs have been identified in patients with carotid atherosclerosis, coronary disease, and stroke [31]. HSP of a major periodontal pathogen, such as $P$. gingivalis (GroEL), was also suggested to be a key molecule linking periodontitis (an infectious disease) with atherosclerosis (an autoimmune disease) [32, 33]. Clonal analysis of the Tcells clearly demonstrated that both human HSP60- and $P$. gingivalis GroEL-reactive T-cell populations were present in the peripheral circulation of patients with atherosclerosis [34]. In addition, $P$ gingivalis can induce platelet aggregation [35], and lipopolysaccharide (LPS) from periodontal pathogens can induce the formation of foam cells $[36,37]$. Therefore, we assessed the potential of periodontal infection in atherosclerosis progression.

\section{Role of Periodontal Infection in Atherosclerosis Progression}

Animal models have been used to examine atherosclerotic pathology caused by periodontopathic bacteria. $P$. gingivalis has been reported by our and other groups to accelerate atherosclerosis in ApoE-KO or apolipoprotein Edeficient spontaneously hyperlipidemic $\left(\mathrm{ApoE}^{\mathrm{shl}}\right)$ mice [3841]. A. actinomycetemcomitans bacteremia also aggravated atherosclerosis in $\mathrm{ApoE}^{\text {shl }}$ mice $[42,43]$. In addition to murine models, rabbits with induced periodontal disease accumulated fatty streaks in the aorta earlier than periodontally healthy animals [44]. P. gingivalis bacteremia also enhanced atherosclerosis in normocholesterolemic and hypercholesteromic pigs [45]. Thus, in addition to the detection of live periodontal pathogens in human atherosclerotic plaques, in vivo experiments in a variety of animal models have corroborated that $P$. gingivalis can enhance atherogenesis.

\section{Endothelial Cell Activation}

Periodontal bacteria and their products can initiate an inflammatory response in the periodontal tissues with systemic consequences. Moreover, bacteria and their products can gain access to the circulation during dental procedures such as scaling and root planning, or even after vigorous home care, especially in patients with periodontitis. Once in the blood stream, bacterial antigens can induce a systemic immune response. Periodontal bacteria in the blood can be carried to distant sites such as the heart tissues freely in the circulation or within circulating cells such as monocytes, neutrophils, or platelets and can initiate pathogenic processes. Tissue or cellular invasion is a key virulence property of many bacterial species. Periodontal pathogens including $P$. gingivalis also adhere to and invade various human vascular cells in culture and animal model $[40,46,47]$. Furthermore, $P$. gingivalis can simulate monocyte adhesion to human umbilical vein endothelial cells [40]. Endothelial activation and increased expression of adhesion molecules and chemokines comprise an initial step in the development of atherosclerotic lesions. A. actinomycetemcomitans infection of ApoE ${ }^{\text {shl }}$ mice resulted in increased expression of intercellular adhesion molecule 1 (ICAM-1), monocyte chemoattractant protein 1 (MCP-1), E-selectin, P-selection, chemokine (C-C motif) ligand 19 (CCL19), CCL21, and C-C chemokine receptor 7 in the aorta [42]. Coculture of human aortic endothelial cells with $P$. gingivalis also increased the expression of ICAM-1, vascular cell adhesion molecule 1 (VCAM-1), MCP-1, and E-selectin by a fimbriae-dependent activation mechanism $[48,49]$. Chemical inhibition of endocytosis blocked the upregulation of MCP-1, indicating the need of bacterial invasion for MCP-1 stimulation [50]. In contrast, stimulation of endothelial cells with LPS, an outer membrane protein, and HSP60 derived from $P$. gingivalis had only a slight stimulatory effect on ICAM-1 and VCAM-1 expression [51].

\section{Toll-Like Receptor- and Nod-Like Receptor-Mediated Responses}

Exposure of periodontal pathogens to endothelial cells may result in increased expression and interaction with Tolllike receptors (TLRs). A. actinomycetemcomitans infection of ApoE $^{\text {shl }}$ mice enhanced mRNA expression of TLR2, TLR4, TLR-9, and nucleotide binding oligomerization domain 1 (NOD-1) in the aorta $[42,43]$. Treatment with the heat-killed 
(H.K.) A. actinomycetemcomitans or A. actinomycetemcomitans LPS also increased TLR4, NOD1, and NOD2 expression. However, the order of atherosclerosis extent was as follows: live A. actinomycetemcomitans $>$ H.K. A. actinomycetemcomitans $>$ A. actinomycetemcomitans LPS. Furthermore, a significant difference in atherosclerotic lesion size was observed between $A$. actinomycetemcomitans-challenged mice and $A$. actinomycetemcomitans LPS-challenged mice. ApoE knockout mice challenged with $P$. gingivalis also expressed TLR2 and TLR4 in aortic tissue [39], while challenge with an invasion-impaired $P$. gingivalis fimbriae-deficient mutant did not upregulate TLRs, suggesting that innate immune recognition of invasive bacteria is a prerequisite for the acceleration of atherosclerosis. In contrast, $\operatorname{ApoE}(-/-)$ and TLR4(-/-) double-knockout mice were markedly more susceptible to atherosclerosis after oral infection with $P$. gingivalis, demonstrating an atheroprotective role for TLR4 in response to $P$. gingivalis infection [52].

\section{Oxidative Stress-Mediated Mechanisms}

Lipid peroxidation plays an important role in many diseases [53]. In particular, low-density lipoprotein (LDL) oxidation may be a key step in the development of atherosclerosis [54]. Oxidation of LDL is essential for its accumulation within the macrophages and for the formation of foam cells, which can upregulate proatherogenic chemokines and adhesion molecules [55] and induce interleukin 6 (IL-6), tumor necrosis factor alpha, and C-reactive protein secretion [56]. A. actinomycetemcomitans infection of $\mathrm{ApoE}^{\text {shl }}$ mice promoted LDL oxidation, as indicated by the marked upregulation of 4-hydroxynoneal, oxidated LDL, and phospholipase A2 in the aorta, ox-LDL, 8-oxo- $2^{\prime}$-deoxyguanosine, and myeloperoxidase serum levels, and aortic expression of nicotinamide adenine dinucleotide phosphate oxidase, caveolin-1, and RAGE [43]. P. gingivalis also increased oxidative modification of LDL $[57,58]$ and rupture of atherosclerotic plaque through induction of MMP [59]. Indeed, patients with periodontitis have increased levels of lipid peroxidation in plasma, saliva, and gingival crevicular fluid [60], and these levels have been correlated with the severity of periodontal disease $[60,61]$. Coincubation of a murine macrophage cell line with $P$. gingivalis in the presence of LDL resulted in the formation of foam cells in a dose-dependent manner [62].

\section{Hyperlipidemia-Induced Atherosclerosis}

CVD remains the leading cause of morbidity and mortality in the Western world. A sedentary lifestyle and Western dietary habits may contribute to this increased risk of CVD development $[63,64]$. For example, consumption of a diet rich in saturated fat is positively associated with elevated plasma lipid levels and a state of subacute chronic inflammation, which are important risk factors promoting both the onset and development of CVD $[65,66]$. Research on a correlation between periodontopathic bacteria and atherosclerosis has been performed using hyperlipidemic animals [38, 39, 45, 67]. Furthermore, a previous study demonstrated that an increase in atherosclerosis is associated with $P$. gingivalis infection in B6.ApoE ${ }^{\text {shl }}$ mice, but not in wild-type mice fed a regular chow diet (RD) [68]. In contrast, $P$. gingivalis injection significantly increased the size and lipid content of atherosclerotic lesions in C57BL/6 mice fed a high fat diet (HFD) [69]. Inoculation of $C$. pneumoniae into C57BL/6 mice fed a HFD also accelerated hypercholesterolemia-induced atherosclerosis [70]. In contrast, chlamydial inoculation into C57BL/6 mice fed the RD did not affect aortic lipid accumulation, although inflammatory changes were induced [71]. Upon infection with $P$. gingivalis, gene expression profiles of the aorta and liver in wild-type mice showed proatherogenic profiles, even in animals fed the RD [68]. Challenge with $P$. gingivalis also induced inflammation associated with elevated serum IL-6, IL-8, and MCP-1 levels, even in C57BL/6 mice fed the RD; however, plaque formation was only slightly elevated. Therefore, the inflammatory response caused by periodontal infection may act in concert with hyperlipidemia to exacerbate atherosclerotic lesion formation.

\section{Prevention of Atherosclerosis by Mucosal Vaccination}

Since several possible mechanisms may be involved in the acceleration of atherosclerosis by periodontal pathogens, the prevention of periodontal infection may be an effective way to reduce the induction of atherosclerosis, as well as periodontitis. Thus, the prevention of periodontitis might be relevant not only for oral, but also for systemic health. Atherosclerosis and inflammation in $\mathrm{ApoE}^{\text {shl }}$ and HFD-fed C57/BL6 mice challenged with $P$. gingivalis were effectively prevented by nasal immunization with the $40 \mathrm{kDa}$ outer membrane protein of $P$. gingivalis $[41,69]$. Furthermore, since HSP60 (GroEL) from $P$. gingivalis can trigger molecules linking infectious periodontitis and autoimmune atherosclerosis $[32,33]$, mucosal administration of a relevant autoantigen is an effective method of attenuating autoimmune disease by inducing an unresponsive state of tolerance [72, 73]. Sublingual immunization with $P$. gingivalis GroEL controlled the acceleration of disease by $P$. gingivalis infection with an increase in the GroEL antibody [74]. Therefore, mucosal vaccination with GroEL may control inflammation and the progression of atherosclerosis due to periodontopathic bacterial infection.

\section{Prevention of Atherosclerosis Using an Anti-Inflammatory Agent}

Previous studies have indicated that early activation of inflammatory mediators in response to a challenge with periodontal pathogens may be associated with periodontitisassociated atherosclerosis [41, 42, 69]. Therefore, oral inflammatory diseases and systemic inflammation caused by periodontal infection may contribute to atherosclerosis. Green tea is a popular drink worldwide, and consumption of green tea has been suggested to prevent the development of a variety of diseases, including diabetes, hypertension, cancer, and cardiovascular diseases [75]. The effects of green tea are 


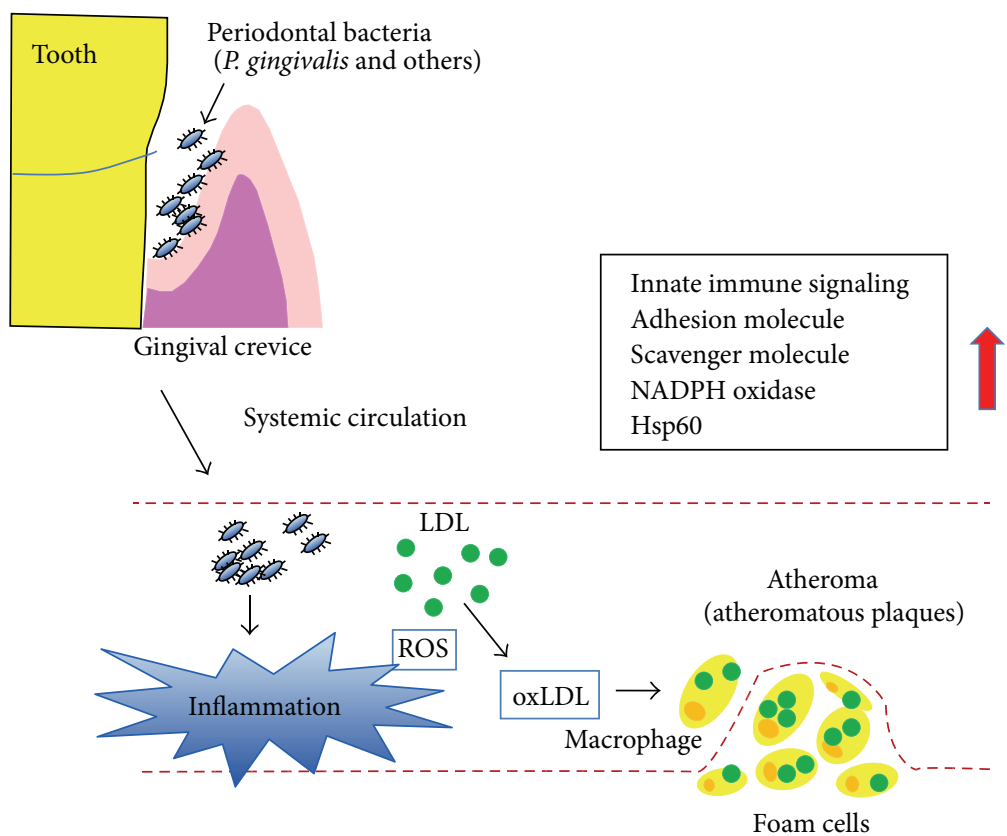

FIGURE 1

attributed to its abundant and biologically active ingredient catechin, or epigallocatechin-3-gallate (EGCG), which has antioxidative [76], anti-inflammatory [77], and antiangiogenic [78] effects. Consumption of EGCG in ApoE knockout mice decreased atherosclerotic lesions, proinflammatory cytokines, and inflammatory- and oxidative stress-related mediators in the serum and aorta induced by $P$. gingivalis [79]. Therefore, previous catechin consumption may be useful for the prevention of pathogen-accelerated atherosclerosis.

\section{Conclusions}

The association between periodontal disease and atherosclerotic cardiovascular disease is supported by a large body of evidence. Periodontal bacteria were shown to accelerate the progression of atherosclerosis in ApoE knockout mice, rabbits, and pigs. Furthermore, observations have suggested that inflammation caused by periodontopathic bacteria may play a synergistic role with other preexisting factors, such as hyperlipidemia, resulting in the development of atherosclerosis. These findings support the hypothesis that a periodontal pathogen is not an independent risk factor but instead acts in concert with hyperlipidemia to exacerbate atherosclerosis lesion formation. However, whether live bacteria are necessary for this process, or if its component alone is sufficient to cause an increase in atherosclerotic damage, remains unclear. In a complex tissue, such as an atherosclerotic lesion, innate signals can originate from several sources and promote atherogenesis through an association with patternrecognition receptors (PRRs). These signals include various extracellular activation cascades and intracellular signaling pathways and lead to effective clearance of infectious agents and induction of inflammatory responses. Since live bacteria can activate multiple PRRs, they may induce a more significant inflammatory response than their components. Finally, the best method to prevent atherosclerosis is by blocking bacterial invasion and bacteria-induced inflammation and lipid peroxidation. Previous results have demonstrated that atherosclerosis and inflammation with lipid peroxidation are accelerated in ApoE-deficient mice after an infection of periodontal bacteria (Figure 1) and that these can be prevented by mucosal immunization with bacterial products or an anti-inflammatory agent.

\section{Disclosure}

The English in this document has been checked by at least two professional editors, both native speakers of English. For a certificate, please see http://www.textcheck.com/certificate/iW3mmS.

\section{Conflict of Interests}

The authors declare that there is no conflict of interests regarding the publication of this paper.

\section{References}

[1] W. J. Loesche and N. S. Grossman, "Periodontal disease as a specific, albeit chronic, infection: diagnosis and treatment," Clinical Microbiology Reviews, vol. 14, no. 4, pp. 727-752, 2001.

[2] B. Burt, "Epidemiology of periodontal diseases," Journal of Periodontology, vol. 76, no. 8, pp. 1406-1419, 2005.

[3] I. Olsen, "Update on bacteraemia related to dental procedures," Transfusion and Apheresis Science, vol. 39, no. 2, pp. 173-178, 2008. 
[4] K. Nakano, H. Nemoto, R. Nomura et al., "Detection of oral bacteria in cardiovascular specimens," Oral Microbiology and Immunology, vol. 24, no. 1, pp. 64-68, 2009.

[5] K. Nakano, H. Inaba, R. Nomura et al., "Distribution of Porphyromonas gingivalis fimA genotypes in cardiovascular specimens from Japanese patients," Oral Microbiology and Immunology, vol. 23, no. 2, pp. 170-172, 2008.

[6] N. Kurihara, Y. Inoue, T. Iwai, M. Umeda, Y. Huang, and I. Ishikawa, "Detection and localization of periodontopathic bacteria in abdominal aortic aneurysms," European Journal of Vascular and Endovascular Surgery, vol. 28, no. 5, pp. 553-558, 2004.

[7] M. Sanz, F. D'aiuto, J. Deanfield, and F. Fernandez-Avilés, "European workshop in periodontal health and cardiovascular disease-scientific evidence on the association between periodontal and cardiovascular diseases: a review of the literature," European Heart Journal Supplement, vol. 12, pp. B3-B12, 2010.

[8] P. B. Lockhart, A. F. Bolger, P. N. Papapanou et al., "Periodontal disease and atherosclerotic vascular disease: does the evidence support an independent association? A scientific statement form the American Heart Association," Circulation, vol. 125, no. 20, pp. 2520-2544, 2012.

[9] T. Dietrich, P. Sharma, C. Walter, P. Weston, and J. Beck, "The epidemiological evidence behind the association between periodontitis and incident atherosclerotic cardiovascular disease," Journal of Clinical Periodontology, vol. 40, no. 4, supplement, pp. S70-S84, 2013.

[10] P. Bouchard, P. Boutouyrie, F. D’aiuto et al., "European workshop in periodontal health and cardiovascular disease consensus document," European Heart Journal Supplement, vol. 12, pp. B13-B22, 2010.

[11] Y. S. Khader, Z. S. M. Albashaireh, and M. A. Alomari, "Periodontal diseases and the risk of coronary heart and cerebrovascular diseases: a meta-analysis," Journal of Periodontology, vol. 75, no. 8, pp. 1046-1053, 2004.

[12] S. J. Janket, A. E. Baird, S. K. Chuang, and J. A. Jones, "Metaanalysis of periodontal disease and risk of coronary heart disease and stroke," Oral Surgery, Oral Medicine, Oral Pathology, Oral Radiology, and Endodontics, vol. 95, no. 5, pp. 559-569, 2003.

[13] J. D. Beck, J. R. Elter, G. Heiss, D. Couper, S. M. Mauriello, and S. Offenbacher, "Relationship of periodontal disease to carotid artery intima-media wall thickness: the atherosclerosis risk in communities (ARIC) study," Arteriosclerosis, Thrombosis, and Vascular Biology, vol. 21, no. 11, pp. 1816-1822, 2001.

[14] J. D. Beck, P. Eke, G. Heiss et al., "Periodontal disease and coronary heart disease: a reappraisal of the exposure," Circulation, vol. 112, no. 1, pp. 19-24, 2005.

[15] B. Holtfreter, K. Empen, S. Glaser et al., "Periodontisis is associated with endothelial dysfunction in a general population: a cross-sectional study," PLoS ONE, vol. 8, no. 12, Article ID e84603, 2013

[16] B. R. Dorn, W. A. Dunn Jr., and A. Progulske-Fox, "Invasion of human coronary artery cells by periodontal pathogens," Infection and Immunity, vol. 67, no. 11, pp. 5792-5798, 1999.

[17] A. Sharma, E. K. Novak, H. T. Sojar, R. T. Swank, H. K. Kuramitsu, and R. J. Genco, "Porphyromonas gingivalis platelet aggregation activity: outer membrane vesicles are potent activators of murine platelets," Oral Microbiology and Immunology, vol. 15, no. 6, pp. 393-396, 2000.

[18] P. Libby, P. M. Ridker, and A. Maseri, "Inflammation and atherosclerosis," Circulation, vol. 105, no. 9, pp. 1135-1143, 2002.
[19] L. Yuan, P. H. Rodrigues, M. Bélanger, W. A. Dunn Jr., and A. Progulske-Fox, "Porphyromonas gingivalis htrA is involved in cellular invasion and in vivo survival," Microbiology, vol. 154, no. 4, pp. 1161-1169, 2008.

[20] P. M. Fives-Taylor, D. H. Meyer, K. P. Mintz, and C. Brissette, "Virulence factors of Actinobacillus actinomycetemcomitans," Periodontology 2000, vol. 20, no. 1, pp. 136-167, 1999.

[21] I. Brook, "The role of anaerobic bacteria in upper respiratory tract and other head and neck infections," Current Infectious Disease Reports, vol. 9, no. 3, pp. 208-217, 2007.

[22] C. G. Daly, D. H. Mitchell, J. E. Highfield, D. E. Grossberg, and D. Stewart, "Bacteremia due to periodontal probing: a clinical and microbiological investigation," Journal of Periodontology, vol. 72, no. 2, pp. 210-214, 2001.

[23] E. Leivadaros, U. van der Velden, S. Bizzarro et al., "A pilot study into measurements of markers of atherosclerosis in periodontitis," Journal of Periodontology, vol. 76, no. 1, pp. 121-128, 2005.

[24] K. Buhlin, M. Hultin, O. Norderyd et al., "Risk factors for atherosclerosis in cases with severe periodontitis," Journal of Clinical Periodontology, vol. 36, no. 7, pp. 541-549, 2009.

[25] Y. Higashi, C. Goto, T. Hidaka et al., "Oral infectioninflammatory pathway, periodontitis, is a risk factor for endothelial dysfunction in patients with coronary artery disease," Atherosclerosis, vol. 206, no. 2, pp. 604-610, 2009.

[26] S. Fedele, W. Sabbah, N. Donos, S. Porter, and F. D’Aiuto, "Common oral mucosal diseases, systemic inflammation, and cardiovascular diseases in a large cross-sectional US survey," The American Heart Journal, vol. 161, no. 2, pp. 344-350, 2011.

[27] J. B. Bartruff, R. A. Yukna, and D. L. Layman, "Outer membrane vesicles from Porphyromonas gingivalis affect the growth and funtion of cultured human gingival fibroblasts and umbilical vein endothelial cells," Journal of Periodontology, vol. 76, no. 6, pp. 972-979, 2005.

[28] R. E. Fitzpatrick, L. C. Wijeyewickrema, and R. N. Pike, “The gingipains: scissors and glue of the periodontal pathogen, Porphyromonas gingivalis," Future Microbiology, vol. 4, no. 4, pp. 471-487, 2009.

[29] J. Oscarsson, M. Karched, B. Thay, C. Chen, and S. Asikainen, "Proinflammatory effect in whole blood by free soluble bacterial components released from planktonic and biofilm cells," $B M C$ Microbiology, vol. 8, article 206, 2008.

[30] A. Kol, G. K. Sukhova, A. H. Lichtman, and P. Libby, "Chlamydial heat shock protein 60 localizes in human atheroma and regulates macrophage tumor necrosis factor- $\alpha$ and matrix metalloproteinase expression," Circulation, vol. 98, no. 4, pp. 300-307, 1998.

[31] K. Mandal, M. Jahangiri, and Q. Xu, "Autoimmunity to heat shock proteins in atherosclerosis," Autoimmunity Reviews, vol. 3, no. 2, pp. 31-37, 2004.

[32] J. I. Choi, S. W. Chung, H. S. Kang et al., "Epitope mapping of Porphyromonas gingivalis heat-shock protein and human heat-shock protein in human atherosclerosis," Journal of Dental Research, vol. 83, no. 12, pp. 936-940, 2004.

[33] J. Choi, S. Y. Lee, K. Kim, and B. K. Choi, "Identification of immunoreactive epitopes of the Porphyromonas gingivalis heat shock protein in periodontitis and atherosclerosis," Journal of Periodontal Research, vol. 46, no. 2, pp. 240-245, 2011.

[34] K. Yamazaki, Y. Ohsawa, H. Itoh et al., "T-cell clonality to Porphyromonas gingivalis and human heat shock protein 60s in patients with atherosclerosis and periodontitis," Oral Microbiology and Immunology, vol. 19, no. 3, pp. 160-167, 2004. 
[35] M. A. Curtis, M. Macey, J. M. Slaney, and G. L. Howells, "Platelet activation by protease I of Porphyromonas gingivalis W83," FEMS Microbiology Letters, vol. 110, no. 2, pp. 167-173, 1993.

[36] H. K. Kuramitsu, M. Qi, I. C. Kang, and W. Chen, "Role for periodontal bacteria in cardiovascular diseases," Annals of periodontology, vol. 6, no. 1, pp. 41-47, 2001.

[37] M. Morishita, W. Ariyoshi, T. Okinaga, M. Usui, K. Nakashima, and T. Nishihara, "A. actinomycetemcomitans LPS enhances foam cell formation induced by LDL," Journal of Dental Research, vol. 92, no. 3, pp. 241-246, 2013.

[38] E. Lalla, I. B. Lamster, M. A. Hofmann et al., "Oral infection with a periodontal pathogen accelerates early atherosclerosis in apolipoprotein E-null mice," Arteriosclerosis, Thrombosis, and Vascular Biology, vol. 23, no. 8, pp. 1405-1411, 2003.

[39] F. C. Gibson III, C. Hong, H.-H. Chou et al., "Innate immune recognition of invasive bacteria accelerates atherosclerosis in apolipoprotein E-deficient mice," Circulation, vol. 109, no. 22, pp. 2801-2806, 2004.

[40] S. Amar, S.-C. Wu, and M. Madan, "Is Porphyromonas gingivalis cell invasion required for atherogenesis? Pharmacotherapeutic implications," Journal of Immunology, vol. 182, no. 3, pp. 15841592, 2009.

[41] Y. Koizumi, T. Kurita-Ochiai, S. Oguchi, and M. Yamamoto, "Nasal immunization with Porphyromonas gingivalis outer membrane protein decreases $P$. gingivalis-induced atherosclerosis and inflammation in spontaneously hyperlipidemic mice," Infection and Immunity, vol. 76, no. 7, pp. 2958-2965, 2008.

[42] T. Zhang, T. Kurita-Ochiai, T. Hashizume, Y. Du, S. Oguchi, and M. Yamamoto, "Aggregatibacter actinomycetemcomitans accelerates atherosclerosis with an increase in atherogenic factors in spontaneously hyperlipidemic mice," FEMS Immunology and Medical Microbiology, vol. 59, no. 2, pp. 143-151, 2010.

[43] R. Jia, T. Kurita-Ochiai, S. Oguchi, and M. Yamamoto, "Periodontal pathogen accelerates lipid peroxidation and atherosclerosis," Journal of Dental Research, vol. 92, pp. 247-252, 2013.

[44] A. Jain, E. L. Batista Jr., C. Serhan, G. L. Stahl, and T. E. van Dyke, "Role for periodontitis in the progression of lipid deposition in an animal model," Infection and Immunity, vol. 71, no. 10, pp. 6012-6018, 2003.

[45] N. Brodala, E. P. Merricks, D. A. Bellinger et al., "Porphyromonas gingivalis bacteremia induces coronary and aortic atherosclerosis in normocholesterolemic and hypercholesterolemic pigs," Arteriosclerosis, Thrombosis, and Vascular Biology, vol. 25, no. 7, pp. 1446-1451, 2005.

[46] A. D. Haffajee and S. S. Socransky, "Microbial etiological agents of destructive periodontal diseases," Periodontology 2000, vol. 5, pp. 78-111, 1994.

[47] R. G. Deshpande, M. Khan, and C. A. Genco, "Invasion strategies of the oral pathogen Porphyromonas gingivalis: implications for cardiovascular disease," Invasion and Metastasis, vol. 18, no. 2, pp. 57-69, 1999.

[48] H.-H. Chou, H. Yumoto, M. Davey et al., "Porphyromonas gingivalis fimbria-dependent activation of inflammatory genes in human aortic endothelial cells," Infection and Immunity, vol. 73, no. 9, pp. 5367-5378, 2005.

[49] Y. Takahashi, M. Davey, H. Yumoto, F. C. Gibson III, and C. A. Genco, "Fimbria-dependent activation of pro-inflammatory molecules in Porphyromonas gingivalis infected human aortic endothelial cells," Cellular Microbiology, vol. 8, no. 5, pp. 738757, 2006.

[50] I.-C. Kang and H. K. Kuramitsu, "Induction of monocyte chemoattractant protein-1 by Porphyromonas gingivalis in human endothelial cells," FEMS Immunology and Medical Microbiology, vol. 34, no. 4, pp. 311-317, 2002.

[51] T. Honda, T. Oda, H. Yoshie, and K. Yamazaki, "Effects of Porphyromonas gingivalis antigens and proinflammatory cytokines on human coronary artery endothelial cells," Oral Microbiology and Immunology, vol. 20, no. 2, pp. 82-88, 2005.

[52] C. Hayashi, G. Papadopulos, C. V. Gudino et al., "Protective role for TLR4 signaling in atherosclerosis progression as revealed by infection with a common oral pathogen," The Journal of Immunology, vol. 189, no. 7, pp. 3681-3688, 2012.

[53] M. K. Logani and R. E. Davies, "Lipid oxidation: biologic effects and antioxidants-a review," Lipids, vol. 15, no. 6, pp. 485-495, 1980.

[54] R. Stocker and J. F. Keaney Jr., "Role of oxidative modifications in atherosclerosis," Physiological Reviews, vol. 84, no. 4, pp. 13811478, 2004.

[55] D. Li, L. Liu, H. Chen, T. Sawamura, and J. L. Mehta, "LOX-1, an oxidized LDL endothelial receptor, induces CD40/CD40L signaling in human coronary artery endothelial cells," Arteriosclerosis, Thrombosis, and Vascular Biology, vol. 23, no. 5, pp. 816-821, 2003.

[56] J. Hulthe and B. Fagerberg, "Circulating oxidized LDL is associated with increased levels of cell-adhesion molecules in clinically healthy 58-year old men (AIR study)," Medical Science Monitor, vol. 8, no. 3, pp. CR148-CR152, 2002.

[57] M. Hashimoto, T. Kadowaki, T. Tsukuba, and K. Yamamoto, "Selective proteolysis of apolipoprotein B-100 by Arg-gingipain mediates atherosclerosis progression accelerated by bacterial exposure," Journal of Biochemistry, vol. 140, no. 5, pp. 713-723, 2006.

[58] T. Bengtsson, H. Karlsson, P. Gunnarsson et al., "The periodontal pathogen Porphyromonas gingivalis cleaves apoB-100 and increases the expression of apoM in LDL in whole blood leading to cell proliferation," Journal of Internal Medicine, vol. 263, no. 5, pp. 558-571, 2008.

[59] Y. Ding, V. J. Uitto, J. Firth et al., "Modulation of host matrix metalloproteinases by bacterial virulence factors relevant in human periodontal diseases," Oral Diseases, vol. 1, no. 4, pp. 279-286, 1995.

[60] F. A. Akalin, E. Baltacioğlu, A. Alver, and E. Karabulut, "Lipid peroxidation levels and total oxidant status in serum, saliva and gingival crevicular fluid in patients with chronic periodontitis," Journal of Clinical Periodontology, vol. 34, no. 7, pp. 558-565, 2007.

[61] A. S. M. Bastos, D. T. Graves, A. P. Loureiro et al., "Lipid peroxidation is associated with the severity of periodontal disease and local inflammatory markers in patients with type 2 diabetes," The , vol. 97, no. 8, pp. E1353-E1362, 2012.

[62] H. Miyakawa, K. Honma, M. Qi, and H. K. Kuramitsu, "Interaction of Porphyromonas gingivalis with low-density lipoproteins: implications for a role for periodontitis in atherosclerosis," Journal of Periodontal Research, vol. 39, no. 1, pp. 1-9, 2004.

[63] B. W. Zagol and R. A. Krasuski, "Effect of motorized scooters on quality of life and cardiovascular risk," The American Journal of Cardiology, vol. 105, no. 5, pp. 672-676, 2010.

[64] L. Qi, M. C. Cornelis, C. Zhang, R. M. van Dam, and F. B. Hu, "Genetic predisposition, western dietary pattern, and the risk of type 2 diabetes in men," The American Journal of Clinical Nutrition, vol. 89, no. 5, pp. 1453-1458, 2009.

[65] P. M. Ridker, "Clinical application of C-reactive protein for cardiovascular disease detection and prevention," Circulation, vol. 107, no. 3, pp. 363-369, 2003. 
[66] M. A. Denke and J. L. Breslow, "Effects of a low fat diet with and without intermittent saturated fat and cholesterol ingestion on plasma lipid, lipoprotein, and apolipoprotein levels in normal volunteers," Journal of Lipid Research, vol. 29, no. 7, pp. 963-969, 1988.

[67] L. Li, E. Messas, E. L. Batista Jr., R. A. Levine, and S. Amar, "Porphyromonas gingivalis infection accelerates the progression of atherosclerosis in a heterozygous apolipoprotein E-deficient murine model," Circulation, vol. 105, no. 7, pp. 861-867, 2002.

[68] T. Maekawa, N. Takahashi, K. Tabeta et al., "Chronic oral infection with Porphyromonas gingivalis accelerates atheroma formation by shifting the lipid profile," PLOS ONE, vol. 6, no. 5, Article ID e20240, 2011.

[69] A. Fukasawa, T. Kurita-Ochiai, T. Hashizume, R. Kobayashi, and M. Yamamoto, "Porphyromonas gingivalis accelerates atherosclerosis in C57BL/6 mice fed a high-fat diet," Immunopharmacology and Immunotoxicology, vol. 34, no. 3, pp. 470476, 2012.

[70] E. Blessing, L. A. Campbell, M. E. Rosenfeld, N. Chough, and C.-C. Kuo, "Chlamydia pneumoniae infection accelerates hyperlipidemia induced atherosclerotic lesion development in C57BL/6J mice," Atherosclerosis, vol. 158, no. 1, pp. 13-17, 2001.

[71] E. Blessing, T. M. Lin, L. A. Campbell, M. E. Rosenfeld, D. Lioyd, and C.-C. Kuo, "Chlamydia pneumoniae induces inflammatory changes in the heart and aorta of normocholesterolemic C57BL/6J mice," Infection and Immunity, vol. 68, no. 8, pp. 4765-4768, 2000.

[72] H. L. Weiner, "Induction of oral tolerance to the acetylcholine receptor for treatment of myasthenia gravis," The Journal of Clinical Investigation, vol. 104, no. 12, pp. 1667-1668, 1999.

[73] C. J. Calder, L. B. Nicholson, and A. D. Dick, "Mechanisms for inducing nasal mucosal tolerance in experimental autoimmune uveoretinitis," Methods, vol. 38, no. 2, pp. 69-76, 2006.

[74] M. Hagiwara, T. Kurita-Ochiai, R. Kobayashi, T. HashizumeTakizawa, K. Yamazaki, and M. Yamamoto, "Sublingual vaccine using GroEL attenuates atherosclerosis," Journal of Dental Research, vol. 93, no. 4, pp. 382-387, 2014.

[75] S. Wolfram, "Effects of green tea and EGCG on cardiovascular and metabolic health," Journal of the American College of Nutrition, vol. 26, no. 4, pp. 373S-388S, 2007.

[76] Q. Feng, T. Kumagai, Y. Torii, Y. Nakamura, T. Osawa, and K. Uchida, "Anticarcinogenic antioxidants as inhibitors against intracellular oxidative stress," Free Radical Research, vol. 35, no. 6, pp. 779-788, 2001.

[77] G. L. Tipoe, T. M. Leung, M. W. Hung, and M. L. Fung, "Green tea polyphenols as an anti-oxidant and anti-inflammatory agent for cardiovascular protection," Cardiovascular and Hematological Disorders Drug Targets, vol. 7, no. 2, pp. 135-144, 2007.

[78] Y. Cao and R. Cao, "Angiogenesis inhibited by drinking tea," Nature, vol. 398, no. 6726, p. 381, 1999.

[79] Y. Cai, T. Kurita-Ochiai, T. Hashizume, and M. Yamamoto, "Green tea epigallocatechin-3-gallate attenuates Porphyromonas gingivalis-induced atherosclerosis," Pathogens and Disease, vol. 67, pp. 76-83, 2013. 


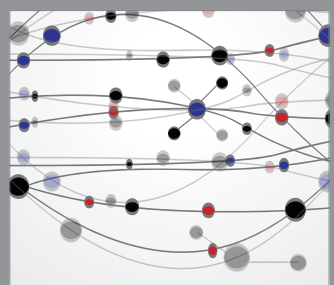

The Scientific World Journal
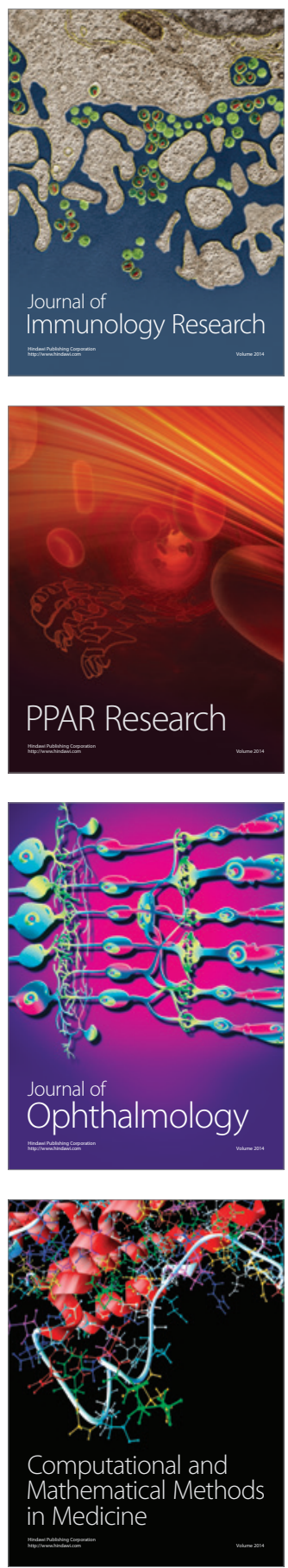

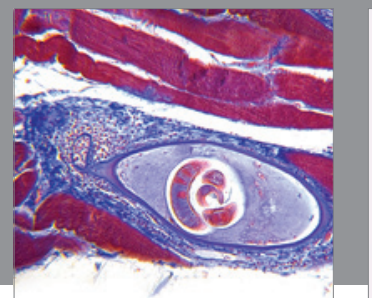

Gastroenterology

Research and Practice
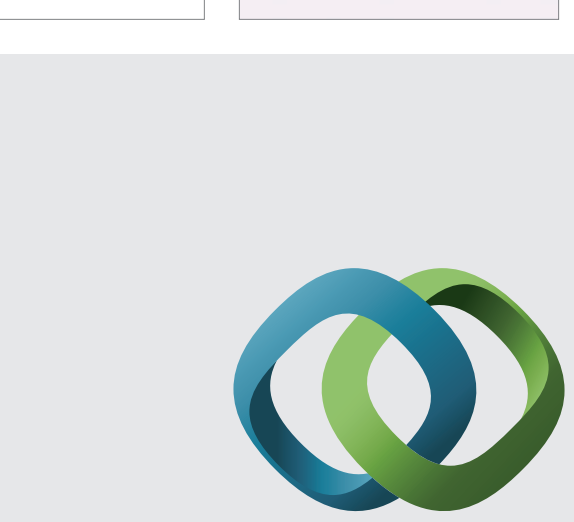

\section{Hindawi}

Submit your manuscripts at

http://www.hindawi.com
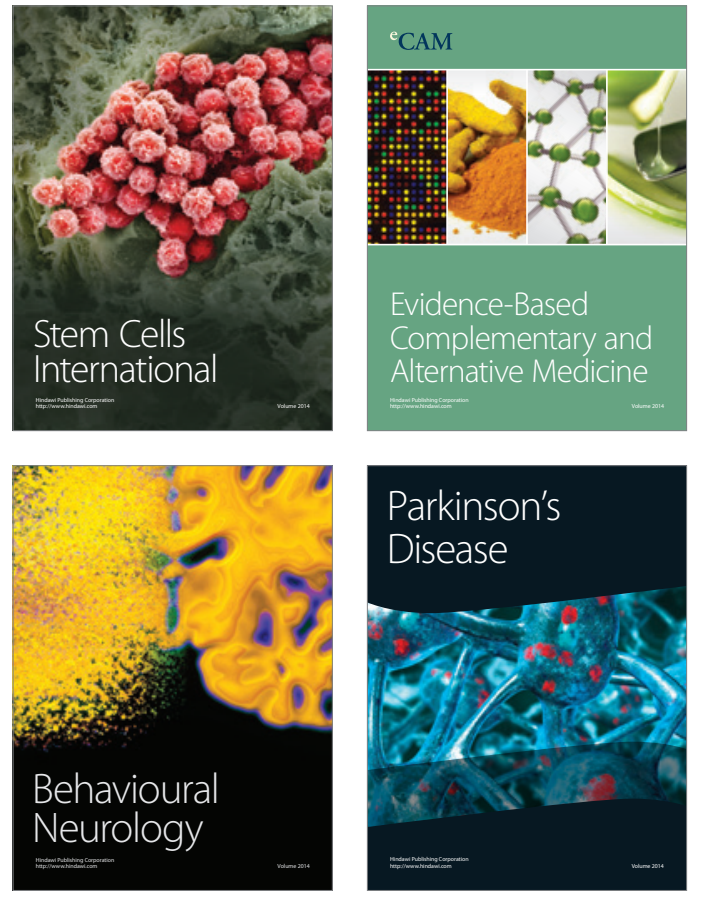
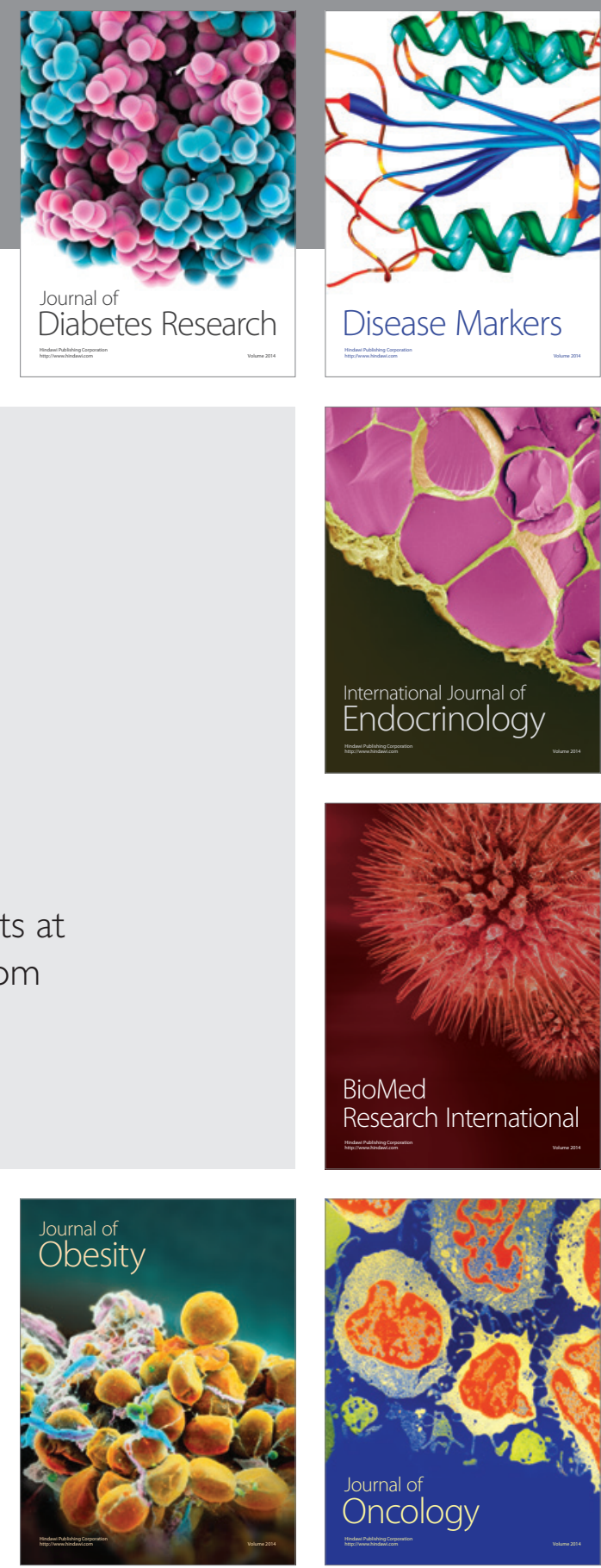

Disease Markers
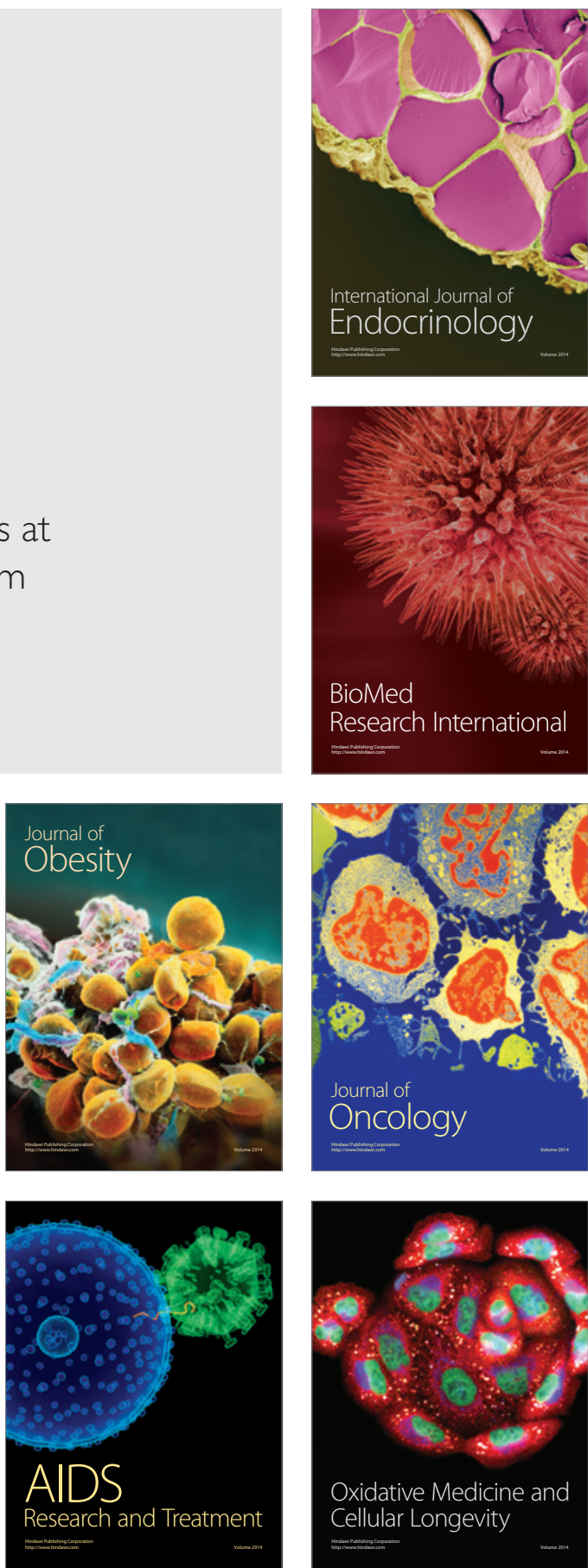